\section{artelogie}

\section{Artelogie}

Recherche sur les arts, le patrimoine et la littérature de l'Amérique latine

2 | 2012

Mexique : espace urbain et résistances artistiques et littéraires face à la « ville générique »

\title{
Vivre sa ville. Guides touristiques et nouveaux récits pour s'approprier Mexico
}

\section{Christine Frérot}

\section{(2) OpenEdition \\ Journals}

Édition électronique

URL : https://journals.openedition.org/artelogie/7733

DOI : 10.4000/artelogie.7733

ISSN : 2115-6395

Éditeur

Association ESCAL

Référence électronique

Christine Frérot, «Vivre sa ville. Guides touristiques et nouveaux récits pour s'approprier Mexico », Artelogie [En ligne], 2 | 2012, mis en ligne le 21 janvier 2012, consulté le 07 janvier 2022. URL : http:// journals.openedition.org/artelogie/7733; DOI : https://doi.org/10.4000/artelogie.7733

Ce document a été généré automatiquement le 7 janvier 2022

Association ESCAL 


\title{
Vivre sa ville. Guides touristiques et nouveaux récits pour s'approprier Mexico
}

\author{
Christine Frérot
}

« Mexico est effectivement la seule ville qui stimule mon imaginaire. J'ai beau avoir vécu aux quatre coins du monde, ça ne marche pas. J'ai bien essayé d'écrire un roman sur Buenos Aires. Mais à quoi bon si Jorge Luis Borges et d'autres le font bien mieux que moi ? Ou Dos Passos avec New York. J'ai un territoire à moi qui est la ville de Mexico, un territoire démesuré avec lequel j'ai trop à faire, qui stimule le désir, l'imagination, la mémoire, le temps, tous ces thèmes que j'aborde depuis mes débuts, sans lesquels je ne pourrais pas écrire. Il faut un sol pour créer et pour moi ce sol c'est Mexico, qu'il tremble, qu'il devienne poussière ou eau. Cette ville est une métamorphose permanente, ce qui est aussi un défi permanent $»$. Carlos Fuentes ${ }^{1}$ 


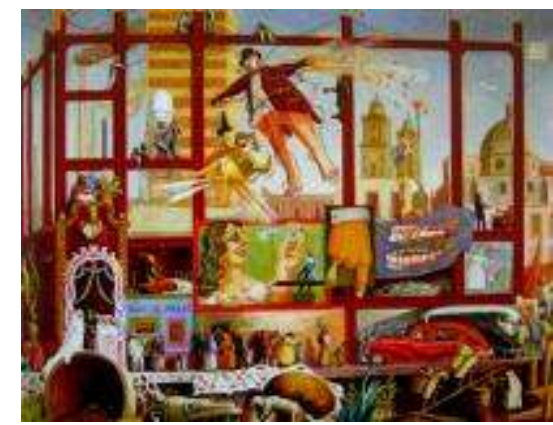

\section{La ville, ses images, ses textes}

$1 \mathrm{Au}$ Mexique, les discours sur la ville et l'imaginaire urbain vont s'accompagner, au tournant des années 2000, de formes d'expression inédites liées à de nouveaux supports éditoriaux. Si l'on examine la production intellectuelle, scientifique et artistique du XXème siècle, il ne fait aucun doute que Mexico n'a cessé d'attirer les créateurs, d'attiser les curiosités, d'alimenter les imaginaires ${ }^{2}$ et de susciter la production d'écrits et d'images. Qu'il s'agisse de fictions littéraires ou anthropologiques, de chroniques, d'analyses historiques ou sociologiques, d'inventaires et de descriptions du patrimoine (édifices et peintures murales), de témoignages photographiques ou picturaux, d'objets de cinéma (la ville comme contexte ou sujet majeur de l'intrigue), Mexico a fasciné les écrivains, les artistes et les universitaires qui ont rendu compte, sous divers aspects, à des niveaux différents et dans plusieurs directions, de ses transformations plurielles, subies ou choisies, au cours du XXème siècle.

2 Commencée avec les Stridentistes ${ }^{3}$, poursuivie par les peintres muralistes et ceux du Taller de la Gráfica Popular, choisie par les photographes, dont Manuel Alvarez Bravo, l'image de la ville change radicalement avec la modernité. L'objet urbain ou industriel s'impose comme thème photographique, ce qu'avaient déjà exploré dès le début du siècle les Frères Casasola, premiers chroniqueurs en images, reporters avant la lettre de la ville de Mexico.

Archivo Casasola, Mexico 1925

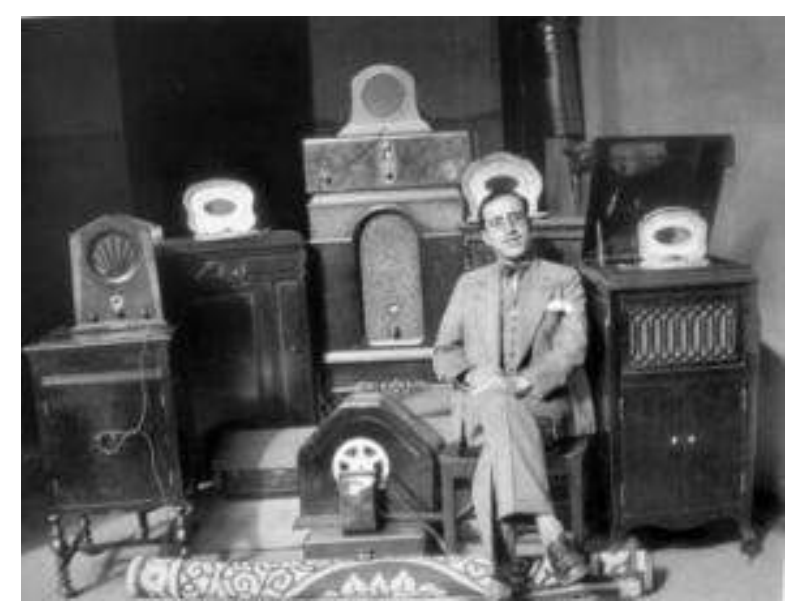


3 Dès le milieu du siècle, tout s'accélère au rythme effréné des changements (accroissement de la population et passage du rural à l'urbain, création d'infrastructures, constructions d'immeubles collectifs et grands édifices officiels, percement de grands axes etc...). Plusieurs visions urbaines, dithyrambiques ou désenchantées, se télescopent; trois peintures de $1949^{4}$ sont révélatrices de l'impact sur les artistes du foudroyant développement urbain. Un tableau emblématique marque triomphalement la naissance de la grande ville et une nouvelle étape dans son évolution (Juan 0'Gorman ${ }^{5}$ ( La ciudad de México). Celui de David A. Siqueiros, Ciudades en evolución, présente une image sombre où la ville semble grignoter inexorablement la nature et étend ses bidonvilles à la périphérie de la capitale. Quant à José Chavez Morado, peintre muraliste très engagé, il propose dans Rio revuelto une évocation caricaturale un peu surréelle, du quotidien, de la folie et du chaos de la grande ville. Mais à l'opposé du progrès urbain encensé par la vision d'o'Gorman, Alfredo Zalce, en mettant l'accent sur ses aspects sordides et tragiques dans sa gravure de 1941, México se transforma en gran ciudad, propose une vision dramatique et désenchantée de la capitale dont le développement chaotique porte en lui la misère, le vol, la faim et la mort. Si l'expansion rapide de la ville interpelle plus ou moins «favorablement ", elle inquiète aussi les artistes et la version la plus terrible est celle peinte en 1947 par Carlos Tejada (La ciudad de México allá por 1970), vision prémonitoire du futur apocalyptique de la capitale mexicaine. La ville rêvée de la modernité s'est transformée en ville honnie, coupable de tous les maux, génératrice de catastrophes.

De nombreux écrivains se confrontent également aux réalités urbaines. Certains d'entre eux, comme Salvador Novo ${ }^{6}$ dans les années 40, exaltent la modernité de Mexico ; Carlos Fuentes publie en 1958 "La plus limpide région", qui est considéré comme le premier roman urbain ${ }^{7}$; à partir des années 60 , Carlos Monsiváis ${ }^{8}$, qui sera l'un des chroniqueurs les plus emblématiques de la capitale au XXème siècle, est en rupture irrévérencieuse avec l'histoire; il critique la politique officielle ${ }^{9}$ et s'intéresse aux figures populaires et à la culture urbaine («contre-culture » et/ou « sous-culture ») qu'ignore la bourgeoisie intellectuelle d'alors. Dès les années 60 , il concevait la chronique comme une obligation - assortie d'un indispensable sens critique ${ }^{10}$ - de se documenter, d'écouter, d'enregistrer, de répertorier les temps nouveaux et les nouveaux espaces, de laisser la parole aux minorités et aux majorités de toute nature qui ne trouvaient pas de place ou de représentativité dans les médias de masse. Les premiers dessinateurs de bande dessinée vont également contribuer à ce climat d'ouverture à la culture populaire. La première B.D. naît en 1948 et deviendra jusqu'à aujourd'hui l'emblème du genre ; La Familia Burrón de Gabriel Vargas (1915-2010), qui aborde la vie quotidienne d'une famille modeste de la capitale, sera un immense succès populaire, durable. Quelques années plus tard, Eduardo del Río, dit Rius, crée deux comics $^{11}$, Los Supermachos en 1964 et Los Agachados (1968 à 1981) qui consacreront auprès d'un large public - même si c'est l'élite intellectuelle urbaine politisée qui les cautionne-, l'engouement pour la vulgarisation humoristique, caricaturale et satyrique des questions sociales et politiques avec des « héros » ou des personnages qui leurs sont familiers. La musique n'échappe pas à cette inspiration nouvelle; le compositeur Chava Flores (1920-1987), auteur de chansons aux titres éloquents comme «El apartamento", "El bautizo de Cheto », «La boda de la vecindad », «El gato viudo », « La esquina de mi barrio " ou «La jardinera de la paleteria », chantées par des interprètes aussi prestigieux que Pedro Infante, Pedro Vargas, José Alfredo Jimenez ou Lola Beltrán, sera à la chronique musicale de la ville ce que Carlos Monsiváis est à la chronique littéraire. 
D'autres images et d'autres textes ouvriront la voie à différents regards sur la ville : dès les années 20, les photographes américain Edward Weston et l'italienne Tina Modotti s'intéressent à la modernité urbaine; plus tard, Elena Poniatowska ${ }^{12}$ commente l'actualité politique; les chroniques actuelles de Cristina Pacheco ${ }^{13}$ et Guadalupe Loaeza ${ }^{14}$ sont publiées régulièrement dans la presse quotidienne comme l'avaient été en son temps les brillantes irruptions et incursions citadines de Jorge Ibargüengoitia ${ }^{15}$ dans le journal L'Excelsior. Et aujourd'hui, ce sont les fictions de jeunes écrivains comme Fabrizio Mejía Madrid, Paco Ignacio Taibo II ou Luis Villoro... qui collent au monde contemporain. Quant à l'étude historique exhaustive, la plus subtile et vivifiante - son auteur écrit d'ailleurs en guise de conclusion que son ouvrage entremêle raisons scientifiques et raisons intimes -, c'est celle de Serge Gruzinski, Histoire de Mexico ${ }^{16}$ dont l'étude s'arrête au milieu des années 90 , après l'explosion des groupes alternatifs de rock.

6 Ces artistes et intellectuels ont ouvert une brèche dans une vision officielle de la ville, surtout pensée pour sa diffusion externe (à l'étranger), en abordant des thèmes politiques ou sociaux plus ou moins conflictuels, plus ou moins censurés. Comme beaucoup d'autres ${ }^{17}$, ils vont alimenter la perception et la conscience des strates qui constituent l'appréhension imaginaire de la capitale et pour certains, inciter le lecteur ou le promeneur à la découverte d'une autre ville, plus « souterraine ", plus marginale, plus vibrante et charnelle, aux antipodes de celle que proposent les récits traditionnels liés au respect et à la valorisation des patrimoines privilégiant l'information historique où est à peine ébauchée l'évocation d'un peuple qui vit, aime, travaille, lutte, crée. Découvrir la «ville invisible» portée par les imaginaires de ses habitants, c'est se souvenir des «Villes invisibles » inventées par Italo Calvino ${ }^{18}$.

7 Réel ou fictionnel, objet de toutes les dramaturgies, scène des plaisirs et des drames, valorisé, encensé, moderne et archaïque, indien et métissé, traditionnel et moderniste, riche ou misérable, public ou privé, marginal, fractionné ou global, un Mexico insolite et surréaliste commence à émerger. Ce regard plus intime, lié au peuple métissé de Mexico et à la valorisation de la culture populaire, précède ce que deviendront au début des années 2000 les nouveaux regards issus d'une autre déambulation urbaine et qui se matérialiseront dans les premiers guides imprimés sur la capitale mexicaine suggérant la découverte d'espaces différents, à pied, à bicyclette ou en voiture. Toujours surprenante, toujours mystérieuse, toujours poétique, décalée, débridée, surprenante et attachante..., même dans le chaos et la violence. Comme le confirme Ruben Gallo ${ }^{19}$ quand il écrit qu'en dépit des périphériques, viaducs et échangeurs qui ont rendu la capitale de plus en plus semblable à d'autres villes moroses, l'extrême vivacité de la culture populaire du centre et d'autres quartiers, berceaux de Superbarrio ${ }^{20}$ et de Chupacabras ${ }^{21}$, a su préserver la métropole du générique.

\section{La ville, nouvel objet de désir}

8 Cette dilatation du «culturellement pensable » va contribuer à forger un regard sur l'inattendu, éduquer à une nouvelle vision de l'objet dans la ville pour le qualifier d'esthétique en marge des canons dominants, décloisonner les conceptions du «beau » et de "l'utile», orienter le regard sur "l'insolite » et faire du »laid» une catégorie valorisée du sensible, en déridant la vision normative par le biais de la poésie urbaine, et en sanctifiant la ville par l'attrait et la séduction de quartiers rénovés (quartiers 
anciens revalorisés et actualisés). Avec la transformation des quartiers, le développement du tourisme urbain intérieur a fait prendre conscience à certains publics de la nécessité d'inventer des outils d'information différents.

Une nouvelle façon de se divertir, de jouir de la vie quotidienne; des habitudes plus internationales; l'impact d'Internet..., dessinent les profils de nouveaux consommateurs, ceux d'un autre public culturel. En dehors des guides sur l'art mural, des années 60, (en anglais, A guide to Modern Mexican Murals, Virgina and Jaime Plenn, édiciones Tolteca, Mexico 1963, ou A guide to Mexican Art, Justino Fernandez, University Of Chicago Press, 1969, pour ne citer que ceux-là, sans oublier le plus récent en espagnol, le très historique et patrimonial Guía retrospectiva de la Ciudad de México, de Rafael Heliodoro Valle, Guillermo Tovar de Teresa y José Lorenzo Cossio ${ }^{22}$ ), le primat du réel, celui de l'actualité sur la valorisante pérennité architecturale et artistique n'est pas encore au cœur des préoccupations sur la ville. En 1998, un ouvrage échappe pourtant à ce constat et exprime déjà un changement dans la consommation culturelle et la curiosité aiguisée pour la culture populaire grâce aux incursions enthousiastes de Carlos Monsiváis. Il s'agit de l'ouvrage d'Armando Jimenez ${ }^{23}$ daté de 1998, Sitios de rompe y rasga en la ciudad de México, Salones de baile . cabarets . billares . teatros ${ }^{24}$. Abondamment illustré, bien que modestement en noir et blanc, ce livre narratif, écrit sur un ton léger, est une mine d'or sur l'histoire et la localisation des salons de danse, des cabarets, des billards et des théâtres de la ville de Mexico. Avec ses photographies des lieux, de danseurs, d'hommes politiques, de musiciens et de compositeurs..., il fait revivre une époque et en consacre une autre, celle-là toujours bien vivante.

Jusque dans les années 2000, on n'édite pas au Mexique de guide touristique sur la capitale ou le pays dans son entier comme il s'en édite de toutes tailles, de tout format, de thèmes divers dans de nombreux pays et dans d'autres langues que l'espagnol depuis de nombreuses années. Les guides touristiques, pour la plupart édités en dehors du Mexique, sont parfois traduits, mais ils sont presque exclusivement conçus pour des visiteurs venant de l'étranger, le tourisme intérieur -des habitants dans leur propre ville-ayant toujours été marginal. Du Guide Bleu (France) au Lonely Planet (Australie) en passant par le Guide du Routard (France) - entre autres -, la diffusion écrite ou iconographique qui est faite pour le touriste, propose/impose une visite calibrée de Mexico, articulée autour d'une version historique consacrée (églises, monuments, musées, art précolombien, etc...), mais aussi d'une vision exotisante, vantant les marchés, l'art populaire, les costumes et les coutumes, sans oublier depuis une quarantaine d'années, avec l'éclatement autant urbanistique que social et économique, l'accent mis sur la violence, vision angoissante de la mégalopole subie autant à l'intérieur qu'exportée à l'extérieur. Quand on clique sur Internet et que l'on recherche les guides sur la ville de Mexico, ce qui saute aux yeux ce sont les avertissements, les mises en garde, les conseils de prudence et ce qu'il faut visiter dans un minimum de temps. La ville y est toujours présentée selon les mêmes schémas et canons (sauf lorsqu'on la présente comme la capitale gay de l'Amérique latine), figée dans une vision aux stéréotypes un peu usés, à la fois séduisante et effrayante, historique et moderne.

11 C'est toute la conception du statut de la ville comme objet touristique qui va changer au cours de ces deux dernières décennies : l'approche de la ville va passer de la tradition patrimoniale $d u$ " tourisme extérieur » à la pratique jouissive du " tourisme intérieur ». La " périphérie » va se déplacer vers le « centre ». Certains vont même passer une fin de semaine dans un Hôtel du centre historique, comme s'ils quittaient la ville et allaient 
ailleurs, pour le visiter à pied - plusieurs rues sont alors piétonnières -, quand il s'est vidé de la majorité de ses voitures, de ses commerçants, de ses bureaucrates et qu'il a été "allégé » de ses innombrables "ambulantes » ${ }^{25}$. Les bouleversements structurels engendrés dans la géographie humaine, sociale et infrastructurelle de Mexico par le tremblement de terre de 1985 , les déplacements de populations qui en découlent, les restaurations et les réhabilitations du centre historique, les modifications de la scène artistique dans la capitale ${ }^{26}$ et la création de nombreux espaces alternatifs ou sur Internet, sont autant d'éléments qui vont peu à peu configurer ce qui depuis vingt ans constitue une nouvelle ville à vivre avec un déplacement des quartiers traditionnellement à la mode ${ }^{27}$ des années 60-70 (Zona Rosa), (Polanco, ouest) et (Coyoacan, sud), vers le Centre ancien (Centro Histórico) et les Colonias Roma et Condesa.

Au cours des années 90 et 2000, l'émergence de quartiers anciens - la Roma de l'Epoque porfirienne (fin du 19ème et début du 20ème) et la Condesa des années 40 - requalifiés pour la jeunesse branchée, les créateurs alternatifs en arts visuels, les artistes aux marges de la culture, les consommateurs de l'éphémère, le loisir autant culinaire (restaurants) que culturel (galeries), la nouvelle culture des cafés et de leurs terrasses..., va consacrer ce que l'ancien ministre de la culture de François Mitterrand, Jack Lang, appelait en son temps (en France dans les années 80) « un nouvel art de vivre culturel». Cette transformation est concomitante avec l'intérêt que vont manifester les décideurs artistiques en organisant des expositions ayant pour thème l'agglomération mexicaine ${ }^{28}$; celle des éditeurs, architectes,

Couvertures des catalogues réalisés à l'occasion de l'exposition "Residual", Mexico 2010
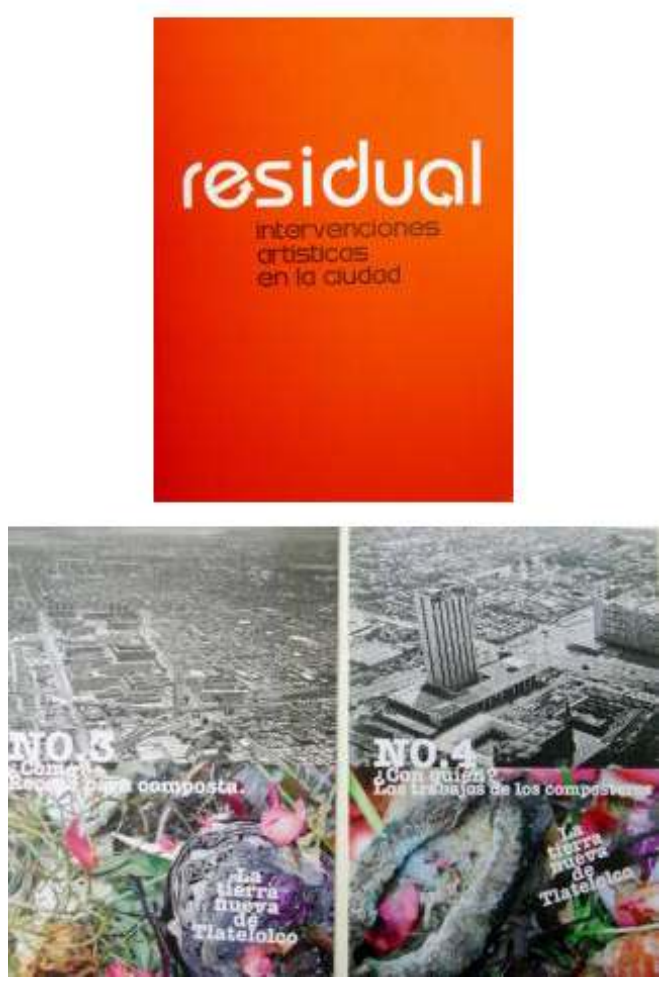

Urbanistes et artistes, en éditant des volumes spécialisés qui font appel à des artistes pour l'illustration photographique comme celui sur la ZMVM en 2000 (Zona 
Metropolitana del Valle de México ${ }^{29}$ et le D.F. en 2001 (ABC DF, Diccionario gráfico de la ciudad de México) ${ }^{30}$ et finalement celle d'un secteur éditorial qui sommeillait, celui des guides sur la ville et le Mexique (editorial Mapas, editorial Travesías) et d'autres maisons d'éditions plus généralistes comme Trilce et Grijalbo.

Un autre facteur dynamisant et non des moindres, est celui de l'implication financière dans des projets éditoriaux (guides) et artistiques (art public) du Gouvernement de la ville (Ciudad de México, Capital en movimiento), ce dernier étant, depuis les années 90, issu de l'élection démocratique. Quatre gouverneurs de gauche ${ }^{31}$ assurent depuis Cuauhtemoc Cardenas en 1997, puis Rosario Robles, Andrès Manuel Lopez Obrador, Alejandro Encinas et Marcelo Ebrard aujourd'hui, une autre politique dans la gestion du District Fédéral qui englobe la ville de Mexico (création de rues piétonnières, installation de circuits de bicyclette, espaces urbains dévolus à la sculpture (sur l'avenue Reforma (entre le Musée d'art moderne et celui d'Anthropologie); réaménagement de l'espace du Ministère des Affaires Etrangères (Relaciones Exteriores) sur l'avenida Juarez...; des opérations culturelles ou artistiques ponctuelles comme celles d'une campagne de sensibilisation écologique et sociale organisée par le Département culturel du D.F., confiée en 2009 à des artistes sur les murs des abris bus des grandes artères, pour ne citer que celle-là.

Si la «meilleure façon de théoriser la ville, c'est de la pratiquer », comme l'écrit Rubén Gallo dans son Anthologie $e^{32}$ en reprenant les idées de Jorge Ibargüengoitia et avant lui, de Salvador $\mathrm{Novo}^{33}$ qui parlait dans «Nueva grandeza mexicana» de "la nécessité d'exercer la ville, c'est-à-dire de la parcourir, de l'arpenter, de l'explorer ", le développement des guides au cours des années 2000 correspond à de nouvelles manières de vivre le rapport à sa ville, à de nouvelles couches sociales et générationnelles, aux groupes qui s'approprient certains quartiers, aux pratiques culturelles et de communications des nouvelles générations Internet, aux rituels et rencontres annuels (Fiesta de la Musica, Corredor cultural de la Roma, Festival del Centro Histórico.....), aux nouveaux musées ou espaces d'exposition (privés) comme Estación Indianilla et Museo del Estanquillo. L'approche de ces guides vise à stimuler l'imagination - les textes de ces ouvrages oscillant parfois entre " fiction » et "réalité " - en proposant des images insolites, en invitant à des découvertes inhabituelles, en mettant l'accent sur un aspect ou un élément de l'espace urbain à priori banal, en mettant en exergue ce qui constitue le tissus intime de la vie de la ville et qui passe pourtant inaperçu..., en bref "à se perdre ", en invitant à trouver du plaisir dans la découverte, à prendre conscience du goût des choses, à entrer plus à fond dans ce que Ruben Gallo appelle «la traversée intérieure des mégalopoles, comparables à une mer sans port $\aleph^{34}$, pour trouver finalement la beauté (cachée) de la vie quotidienne, la rendre visible. Leur rédaction fait appel à des écrivains et à des artistes pour la photographie, d'où la qualité de ces ouvrages qui visent à nous dévoiler les «secrets " et les « mystères » de Mexico, « ville invisible » rendue visible.

\section{Les revues culturelles}

Comme nous venons de le voir, le terrain a été préparé, et la prise de conscience accélérée des artistes, des écrivains et des éditeurs au cours des années 90 et 2000 a ouvert la voie au désir et à la nécessité d'un autre regard sur la mégalopole, une envie de l'appréhender avec d'autres symboles, d'être fasciné par d'autres images et d'autres 
représentations urbaines afin de minimiser les clivages entre culture populaire et culture savante, de valoriser, voire d'officialiser les marges culturelles. Parallèlement aux guides, plusieurs revues culturelles ${ }^{35}$ ont vu le jour au cours de la première décade s'adressant aux mêmes publics que ceux qui vont fréquenter les « nouveaux » quartiers branchés : de même que de nouveaux lieux de diffusion artistique, d'espaces alternatifs qui en seront les relais (comme les Casas vecinas), les revues mensuelles vont apparaître, spécifiquement axées sur les arts, la culture, les sorties, la mode, la musique, le cinéma..., sur l'actualité, pour un public jeune, branché et consommateur comme «CHILANGO » DF no hay nadie como tú $»^{36}$; plus culturelles, qui abordent les sujets de manière approfondie, comme «LA TEMPESTAD $»^{37}$ (qui diffuse un petit guide de Mexico -sorties culturelles, restaurants- séparément avec la revue) et «ALGARABÍA revista que genera adicción ».

Couvertures des revues "Chilango" et "Algarabía"

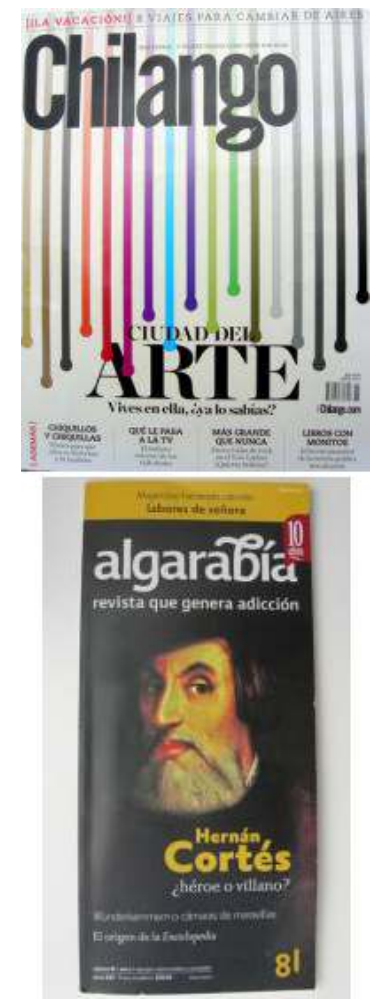

17 Quant au Gouvernement de La Ville (Département des Affaires Culturelles), il contribue à la diffusion de l'information culturelle en publiant un guide mensuel : «CHILANGUÍA, la ciudad de la cultura", publication qui donne des informations partielles sur les activités culturelles (théâtre, cinéma, expositions) avec un petit agenda (Cartelera mensual). Il y a aussi les hebdomadaires, parfois distribués gratuitement, comme « La semana de FRENTE » qui vont compléter de manière ludique et sérieusement informée l'hebdomadaire " TIEMPO LIBRE » (sorte d'Officiel des Spectacles) qui ne s'est jamais amélioré dans le temps et reste très incomplet, désuet dans sa maquette et confus en dépit de son ambition d'être à peu près exhaustif ${ }^{38}$. 
Couverture de la revue "Frente"

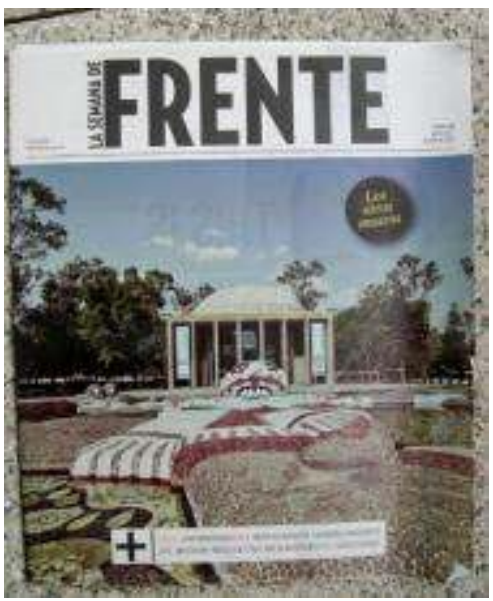

\section{Les guides}

Les trois guides que nous avons choisi de commenter plus largement s'inscrivent dans le développement de ces nouveaux discours et récits sur la ville, en dehors de la place qu'y occupe la littérature actuelle dont nous ne parlerons pas ici. Différents dans la forme, le format et le fond, ils sont chacun les héritiers de cette façon plus libre de chercher et trouver une autre ville dans la ville, et de vivre sa ville. Nous irons du moins conventionnel au plus « classique » pour les trois premiers et terminerons notre inventaire par un guide de petit format, Style Map.

\section{Citambulos. Guía de asombros Ciudad de México. El transcurrir de lo insólito}

19 Auteurs : Ana Alvarez, Valentina Rojas Loa, Christian Von Wissel.Essai de Peter Krieger. Photographies en noir et blanc et couleurs.Editorial Océano, Mexico 2006, 388pages, versions espagnole et anglaise (épuisé en langue espagnole). 
Couvertures du guide "Citámbulos"

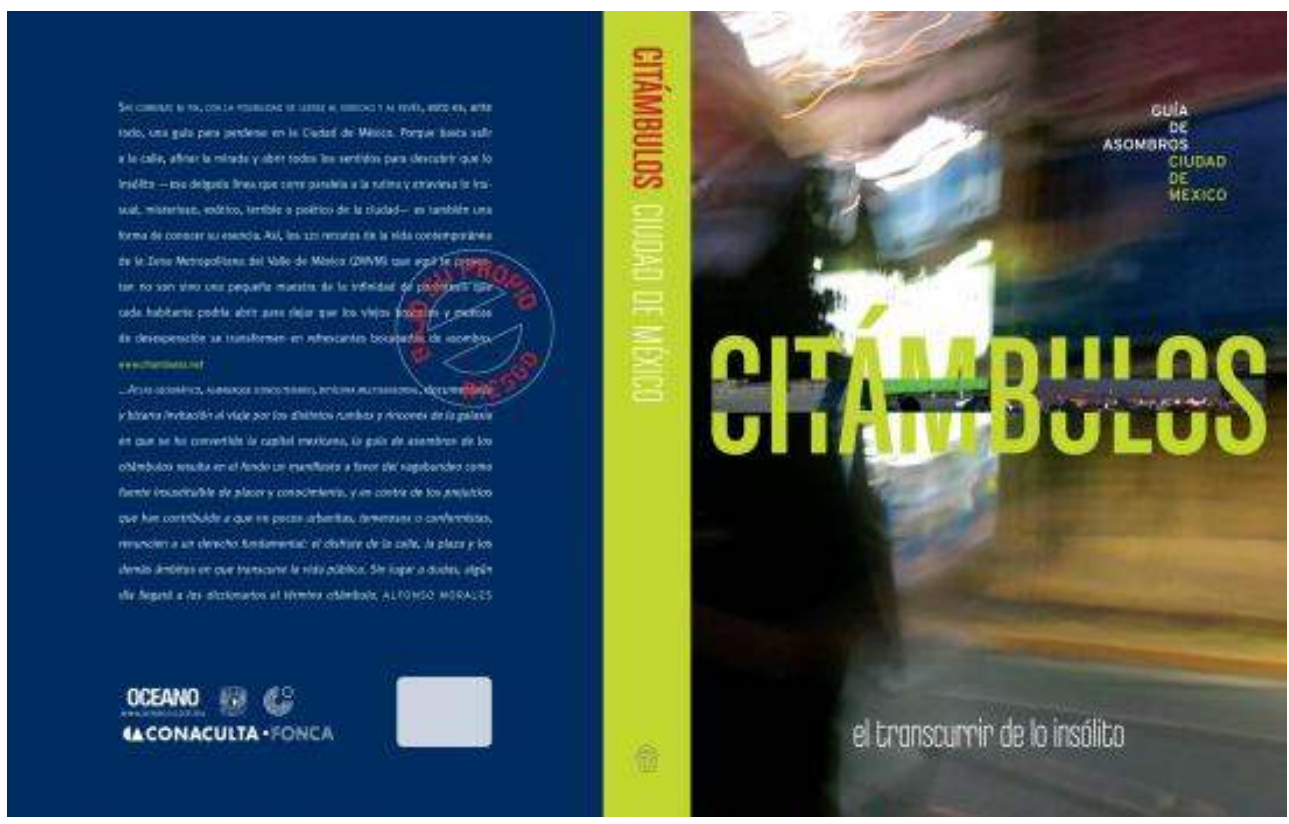

"Sans début ni fin ", le guide brosse avec un vrai brio littéraire cent vingt et un portraits de la vie contemporaine de la ZMVM (Zona metropolitana del valle de México), elle-même découpée en cinq zones : CENTRO HISTÓRICO (centre historique), CIUDAD INTERIOR (ville intérieure), ORIENTE (orient), NORTE (nord), SUR (sud), PONIENTE (ponant). Dans l'introduction intitulée " ¿Qué es?», il se définit ainsi ${ }^{39}$ : Livre, atlas, manifeste, almanach, inventaire, objet inclassable et hétérogène comme la ville dont il parle, c'est avant tout un guide pour se perdre dans la ville de Mexico. Parce qu'il suffit de descendre dans la rue, affiner son regard et ouvrir tous les sens pour découvrir que l'insolitecette ligne étroite qui est parallèle à la routine et traverse ce qui est inhabituel, mystérieux, exotique, terrible ou poétique de la ville - est aussi une façon de connaître son essence. Ce guide est avant tout une invitation à abandonner le fauteuil de lecture, à déambuler dans la ville et à se convertir en citámbulo, en permettant aux anciens bâillements et grimaces de désespoir de se transformer en bouffées rafraîchissantes d'étonnement.

21 La place des images y est essentielle. Non attendues, non convenues, insolites, réalisées par différents photographes et de formats divers (fragmentées, verticales, horizontales, en détail ou répétées), elles occupent la moitié de l'édition.

22 La liberté offerte d'emblée au lecteur est servie par une maquette aérée où chaque thème est développé autour d'une image ou plusieurs, et d'un texte qui prend des formes calligraphiques et littéraires différentes (entrevue, listes, fictions, citations de poème, récit, histoire...). Visuellement, un découpage un peu cinématographique de l'ensemble évoque une sorte de "story-board", où les photographies de détails abondent. Le montage de base de la maquette, autour d'un texte et d'une photographie, n'est pas systématique et laisse alterner des découpes iconographiques qui ont un autre rapport au texte. Chaque sujet porte un numéro accompagné du sigle de la zone dans laquelle se trouve ce dont on parle.

23 Deux index sont situés à la fin de l'ouvrage. Dans le premier, alphabétique, quelques exemple : pour la zone CENTRO HISTÓRICO «El rincón del ahorcado 01 CTR » (le coin du pendu), «El ladrón devoto 02 CTR » (le voleur dévôt), " Dónde hacer sus necesidades 06 CTR » (où faire ses besoins); dans la zone CIUDAD INTERIOR, «Gota a gota 05 INT » 
(goutte à goutte) ou « E.U. vende territorio mexicano a México 14 INT » (Etats-Unis vendent territoire mexicain au Mexique), «Venenosa sensualidad 15 INT » (sensualité vénéneuse) ; dans la zone OESTE, «Japonesque 04 OTE » ou « Paso entre la vida y la muerte 06 OTE » (passage entre la vie et la mort); pour la zone NORTE, des titres comme «American way of belief 04 NTE» (façon américaine de croire), ou " Navegantes subterráneos 01 NTE » (navigants souterrains); dans la zone SUR «Caronte trajinero 17 SUR » (transporteur ambulant) ou « Arquitectura de vanguardia 13 SUR » (architecture d'avant-garde); dans la zone PONIENTE «Auxilio vial 01 PTE » (auxiliaire de la voie publique), « La inevitable inquilina 16 PTE » (l'inévitable locataire), « 1999 perros 20 PTE » (1999 chiens)... De petites vignettes minuscules accompagnent les textes en bas de page (homme qui marche, bicyclette, voiture, charrette à cheval...).

24 Chaque texte se réfère à un lieu, un personnage, un monument, une activité... dont l'adresse et les conditions d'accès sont indiquées en bas de page. Pour reprendre les exemples cités plus haut, , $E l$ rincón del ahorcado » se réfère à un bar clandestin ; « $E l$ ladrón devoto » à " La Vierge des voleurs »; « Dónde hacer sus necesidades », donne la liste des WC publics de Mexico; "Gota a gota », parle des fabriquants de glace ; «E.U. vende territorio mexicano a México » se réfère au cimetière militaire des Etats-Unis ; " Japonesque ", c'est l'Eglise en forme de pagode ; "Paso entre la vida y la muerte », la sculpture du Christ qui se trouve sur un pont piétonnier enjambant une autoroute; "American way of belief ", le Temple mormon; "Auxilio vial », entrevue d'un policier qui assure la circulation automobile; "La inevitable inquilina », ce sont les araignées domestiques et «1999 perros », le refuge pour chiens. Ces textes renvoient à d'autres entrées, indiquées en fin de texte.

De l'humour, de l'ironie, de la curiosité, de l'imagination, de la pertinence, sans jamais tomber dans le scabreux ou l'horreur, façonnent des textes savoureux et originaux dans leur manière très personnelle d'appréhender les réalités, dans leur rédaction à la fois légère et précise, où la dimension littéraire affleure à chaque instant, caractéristiques qui rendent la lecture vraiment passionnante, de surprise en surprise, de découverte en découverte. Emaillée de suggestions insolites, la lecture est sans cesse sollicitée par les images pour découvrir des réalités surprenantes et non conventionnelles. Le terme " touriste " perd avec ce texte la connotation péjorative qu'il peut avoir quelquefois et l'observateur que l'on est au départ devient découvreur, archéologue et arpenteur.

L'autre index est analytique. Il reprend, par ordre alphabétique, les thèmes génériques. Pour n'en citer que quelques-uns : agriculture, arts plastiques, apocalypse, catastrophe, dévotion, eau, football, homosexuels, lutte libre, microcosme, magie, musées, périphérie, synagogue, textures, volcans, vendeurs ambulants, violence, water-closets, zoo..., à l'intérieur desquels sont développés les sous-thèmes présents dans l'ouvrage.

L'imbrication des sujets entre eux, les renvois thématiques, les cartes, les plans, les lettres, les numéros... tout cela semble complexe et compliqué au premier abord. En réalité, il s'agit d'une construction ludique, que l'on pourrait rattacher à l'influence de la culture visuelle et comportementale d'Internet (emboîtement ou mise en abyme des sujets), qui enrichit la perception sensible de la ville et crée une trame à la fois réelle et imaginaire. Dans le chapitre «Como funciona » (Comment fonctionne-t-il), les auteurs nous préviennent qu'il ne s'agit pas d'un seul guide, mais de trois guides en un qui sont «tous consacrés à l'habitant de cette ville à qui l'on propose de choisir l'une des possibilités suivantes (ce qui, bien entendu, n'exclut pas qu'il puisse en explorer d'autres) : 
it se servir du premier guide comme de n'importe quel autre: six zones couvrent toute la surface urbaine de la Zone Métropolitaine de la Vallée de Mexico (ZMVM) et dans chacune de ces zones, on pourra découvrir des itinéraires intégrés par lieux géographiquement proches. L'habitant choisira l'un d'eux, le parcourra, prendra des photos de ses lieux favoris et les montrera à son voisin. Le second guide trace des chemins qui surprennent par les liens qui s'établissent entre des zones très distinctes. Ainsi, à la fin de chaque description de lieu, il existe des portes qui conduisent à un ou plusieurs endroits qui, bien qu'éloignés géographiquement, sont connectés thématiquement. Le troisième guide est plutôt un métaguide, c'est-à-dire une invitation à aller plus loin de ce que laisse une relation seulement intuitive de la ville. C'est ainsi qu'une personne qui y vit, mais surtout l'étudie, ouvre des parenthèses dans les parenthèses que nous avons suggérées icit ${ }^{40}$.

\section{2. ¿A dónde váis monsiváis? Guía del DF de Carlos Monsiváis}

Textes de Carlos Monsiváis et prologue de Fabrizio Mejía Madrid. Editores Déborah Holtz et Juan Carlos Mena avec la collaboration de Laura Emilia Pacheco.Editions Trilce et Grijalbo, avec le soutien de la Commission du Bicentenaire du Gouvernement du District Fédéral.Illustrations en noir et blanc, 358 pages, Mexico 2010. (Dans une pochette de la deuxième de couverture est annexé un plan des quartiers du centre de Mexico, manipulable de façon indépendante).

Couverture du guide "¿A dónde váis Monsiváis ?"



La spécificité de ce guide au format étroit et allongé est qu'il est entièrement construit à partir d'un choix de fragments de nombreux textes de l'écrivain mexicain Carlos Monsiváis (1938-2010). Une approche originale liée aux pas, aux déambulations, aux goûts, aux émotions et aux choix esthétiques de celui que Sergio Gonzalez Rodríguez (p. 308) appelle « el caminante de la ciudad ${ }^{41} »$ et qui a été le chroniqueur-vedette de la ville de Mexico pendant presque toute la seconde moitié du XXème siècle. En quatrième de couverture, nous sommes prévenus par l'affirmation suivante: A donde vais, Monsiváis?", primera guía intelectual del Distrito Federal. Une carte à déplier, autonome, des zones centrales de la ville est placée dans une pochette de la deuxième de 
couverture. Cinq zones ont été délimitées avec trois couleurs et portent un numéro qui correspond à un lieu et à la page qui le concerne dans le guide. Dans son Autobiografía publiée en 1966, l'écrivain journaliste, critique et essayiste auteur de milliers d'articles et de chroniques, de nombreux livres, idolâtré par les intellectuels et les artistes du D.F., exaltait ainsi la ville dont il était véritablement amoureux ${ }^{42}$ : Les leçons ne viennent pas de la contemplation des montagnes, des arbres ou des troupeaux, mais dans la maîtrise des feux de croisement et des avenues.

31 En dehors des extraits de textes de Carlos Monsiváis, d'autres interventions émaillent l'ouvrage. Témoignages de ses amis, comptes-rendus d'entrevues avec l'auteur, anecdotes et aventures partagées à Mexico ou à l'étranger, qui contribuent à rendre ce guide vivant et à mettre en exergue la personnalité de celui qui vous propose de visiter « sa » ville. Parmi eux, on peut citer les écrivains, Carlos Fuentes, Sergio Pitol, Rafaël et José María Perez Gay, José Emilio Pacheco ou Hugo Gutierrez Vega ; des dessinateurs et caricaturistes comme Rafaël Barajas dit «El Fisgón» et Rius (Eduardo del Río); des journalistes, Braulio Peralta (La Jornada); des acteurs, Claudio Obregón ; des artistes, José Luis Cuevas; des essayistes de théâtre, José Luis Ibañez; des politiciens comme Porfirio Muñoz Ledo etc... Une pléiade de contributions d'intellectuels et d'artistes connus valorisent le guide, lui donnent une légitimité supplémentaire. Dans son prologue, l'écrivain Fabrizio Mejía Madrid justifie le choix de celui qui s'est intéressé à culture populaire et dont le lien inextricable avec la ville fait que l'on ne peut parler de Mexico sans Monsiváis (p. 15) : Monsiváis est la ville de Mexico, les lignes de sa main sont un Guide Rojí. La ville de Mexico n'existerait pas sans Monsiváis, c'est lui qui la magnifie, la rend possible, lui voit le côté citoyen, même au milieu des ruines du tremblement de terre de $1985^{43}$.

32 Après l'introduction et quelques extraits de textes de Monsiváis sur Mexico, le guide aborde les quartiers (cinq zones englobant plusieurs quartiers et monuments) abordés par l'auteur. Les textes du chroniqueur sur des lieux très précis et souvent emblématiques comme La Plaza Garibaldi, la Lagunilla, el California Dancing Club, Tlatelolco, San Angel, Ixtapalapa ou la Ciudad Universitaria, sont entrecoupés d'autres textes (des fragments également) des écrivains ou autres intellectuels cités plus haut. Ils sont accompagnés d'une description, non signée (équipe rédactionnelle du guide) du lieu et de l'adresse et de la description succincte d'un café ou d'un restaurant si cela s'impose. C'est donc une découverte de la ville que l'on doit aux coups de cœur de Monsiváis, à un pas à pas en compagnie de ses commentaires, à son regard critique, à son humour irrévérencieux, à ses préférences pour «l'anti-culture » ou les cultures marginales.

Une autre des particularités de ce guide, c'est son iconographie qui veut « coller » à la vie quotidienne de Mexico. Ce n'est pas une belle image de la ville que de guide veut nous donner, sinon une vision des contrastes, du chaos, de l'insolite, de l'activité grouillante au jour le jour. Ce qui compte surtout dans l'illustration en majorité polychrome, ce ne sont pas les monuments qui intéressaient peu Monsiváis (quelques détails cependant), mais les figures de quartier, les hommes, les femmes, les enfants, les ouvriers, les employés, les musiciens, les petits vendeurs de rue, les écolières en uniforme, dans leurs activités journalières ; mais aussi les enseignes, les évènements emblématiques, avec des photographies en noir et blanc (1968 par exemple); et bien entendu les photographies de chats (Carlos Monsiváis les adorait). Ce qui importe, ce sont les contrastes entre modernité (gratte-ciels) et traditions (Mariachis), avec un humour souvent décalé et décapant, présent à chaque page. 


\section{Nueva guía del centro Histórico de México}

\section{Couverture du guide "Centro histórico de México"}

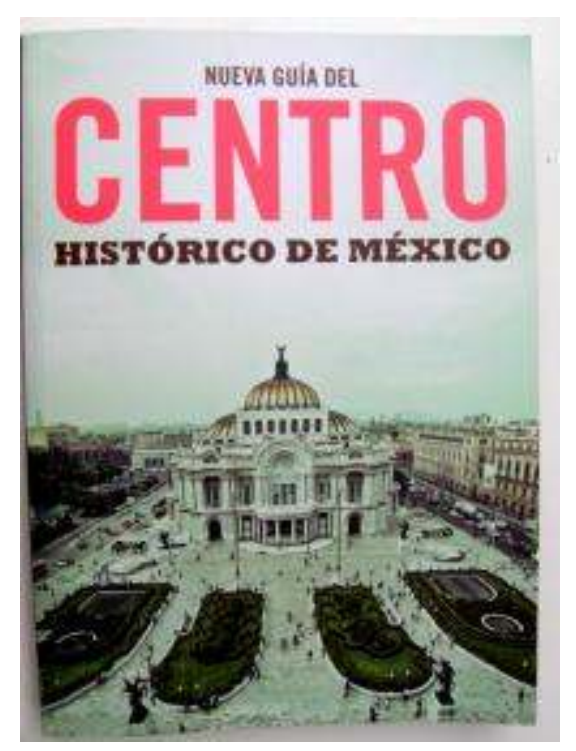

Dans une ville de plus de vingt millions d'habitants, le Centro Histórico dispose d'un patrimoine architectural exceptionnel pour l'Amérique latine. C'est à la suite de la découverte en 1980 des ruines du Templo Mayor que le périmètre du centre historique est officiellement déclaré «zone de monuments historiques" par le Gouvernement fédéral mexicain (superficie de $9 \mathrm{~km}^{2}$ ). Depuis 1987, ce centre est classé patrimoine mondial par l'Unesco. Un accord sera signé en 2001 entre le privé (Carlos Slim, entrepreneur mexicain, première fortune au monde), le Gouvernement fédéral et la Mairie pour la réhabilitation du centre historique : achat d'immeubles et rénovation pour la location à des intellectuels, des artistes ou des jeunes cadres; réfection des trottoirs; refonte du mobilier urbain; création d'un corps de police spécial pour la sécurité touristique ; évacuation des vendeurs ambulants ${ }^{44}$; aménagement de places et piétonisation de rues...., tous ces aménagements concernant une partie du Centro Histórico et notamment ce que l'on appelle le «corridor cultural $»^{45}$. Malgré ces rénovations, l'habitat précaire et insalubre représente environ un tiers du parc de logements et les actions dans le domaine social ne progressent pas vraiment ${ }^{46}$.

Si le constat est socialement mitigé sur les effets de la rénovation du centre historique, il n'en reste pas moins que le pouvoir d'attraction, pour un séjour temporaire plutôt qu'un habitat pérenne, reste fort et que la dimension culturelle ne cesse de se développer. Dans son introduction, l'historien Álfonso Álfaro met l'accent sur la richesse culturelle de cette ville qui associe trois cultures ${ }^{47}$ superposées : Les sédiments du temps ont donné au centre, à la ville et au pays, une spécificité : ils en ont fait un creuset qui réussit à intégrer les présences emblématiques des multiples vecteurs culturels du pays, passés et 
présents, réels et imaginaires, actifs et latents. L'architecte baroque le plus visionnaire n'aurait pu imaginer un dispositif spatial plus efficace pour représenter une société hétérogène à la recherche d'harmonie, une multitude bigarrée de citoyens avides de se construire en tant que nation.

Le guide a été présenté à la presse le 18 février 2011 par le maire de Mexico, Marcelo Ebrard en tant qu'édition du Gouvernement du District Fédéral ${ }^{48}$. Ce Nueva Guía, est un outil grâce auquel on cherche à susciter l'affluence des visiteurs vers les sept zones qui divisent cette partie de la capitale ${ }^{49}$, la sécurité y étant rétablie, selon son maire. Il souligne également que le Centre historique est la pièce maîtresse de l'identité et que la seule façon de consolider les communautés est d'avoir des lieux à visiter de meilleure qualité, dans lesquels on peut vivre et où l'on réalise de nombreuses activités. Le Centre historique est aussi considéré comme le siège d'un processus de réinvention urbaine, dans laquelle la clef fondamentale c'est l'espace public. Il faut que les gens comprennent que c'est un espace vivant et pluriel (...) Le guide est un outil social, culturel et éducatif, pour que les touristes et les visiteurs se rapprochent de manière plus intégrale du Centre historique (Inti Muñoz ${ }^{50}$ ). Le guide CENTRO est donc plus qu'un guide touristique, c'est l'objet d'un enjeu important d'abord politique, puis culturel et social pour le Gouvernement du District Fédéral qui s'y est impliqué financièrement et médiatiquement.

Comment est construit ce guide ? La ville est découpée en sept « Zonas » et chaque zone porte un titre qui détermine sa position géographique dans le centre historique. Dans chaque zone, des quartiers (avec titres et numéros) à l'intérieur desquels sont déclinés les lieux d'intérêt. Prenons un exemple: La Zona 1. Pour commencer, chaque zone dispose d'un plan très clair occupant la page de droite avec numérotation des lieux d'intérêt et parfois des images en trois dimensions des bâtiments, et sur la page de gauche, la liste de ces lieux avec leur numéro correspondant. Chaque zone est assortie d'un texte d'écrivain. Quand il se réfère à un lieu, qu'il s'agisse d'un lieu de passage, de manifestations, de réunions publiques ou de promenade..., comme l'est par exemple le Zócalo, ses descriptions ont une légèreté formelle, ne sont pas enfermées dans un carcan de précisions historiques qui pourraient être un peu ennuyeuses; elles autorisent une liberté de ton, des anecdotes, des opinions personnelles, et permettent au lecteur d'avoir la sensation physique de pénétrer dans le lieu. Les courtes descriptions de monuments, de boutiques ou de restaurants (non signées) partagent la même humeur rédactionnelle. Il s'agit vraiment de sentir, de voir, d'écouter au rythme de celui qui raconte. On est loin d'une description savante, historique et précise. C'est plutôt la température de la ville, une invitation à s'y jeter corps et âme, tous les sens en alerte, à se plonger dans son bouillonnement, ses surprises. Pour la vivre au quotidien, l'écouter, la goûter, la savourer et enfin, la dévorer.

39 Dans la Zona 1, le premier texte est consacré au cœur de la ville, le Zócalo. Dans « Así está el Zócalo", Fabrizio Mejía Madrid écrit (p. 34) $)^{51}$ : Le Zócalo, c'est là que tout a commencé, c'est le big bang de béton. C'est à partir de là, où nous supposons que l'aigle s'est posé sur un cactus avec un serpent dans son bec, que le pays entier s'est étendu, comme un tapis rouge. Le Zócalo a été marché, jardin, échafaudage, table de jeu d'échecs, plat pour la brioche des Rois, cinéma, musée nomade, clinique avec mammographies gratis, motel d'instituteurs, air pour faire voler des cerfs-volants, vitrine de vente de pastilles de peau de serpent, piste de patinage, musée de photographies d'enfants avec des éléphants, cimetière avec des offrandes aux petits défunts, salle de concerts, théâtre où le meilleur spectacle est la multitude elle-même qui se 
regarde dans son immensité. (...) Le Zócalo est la définition de chilango : on peut tout y mettre, rien n'y est en trop.

Il est suivi d'un chapitre intitulé "Sitios de intérès ». On y retrouve, sur un petit plan avec images en trois $\mathrm{D}$, numéros et noms des bâtiments, les lieux qui seront abordés dans ce chapitre. Une rubrique attire notre attention. Intitulée « Gente del barrio », (les gens du quartier), elle s'intéresse à une figure locale, en l'occurrence pour la Zona 1, «El huehuetero » (le danseur et joueur de huehuetl) 52 , avec une photographie pleine page, ce qui donne une vie réelle au quartier concerné. On trouve ensuite les rubriques "Cantinas, cafés, restaurantes", "Vida nocturna ", "Compras ", qui est la partie réellement "guide», avec adresses et téléphones, et enfin, un lieu est lui aussi mis en exergue et accompagné d'un texte, comme «Calle Madero » de Jorge F. Hernandez. Ce n'est que dans les deux dernières pages que l'on trouve une liste d'hôtels du Centro Histórico.

41 D'une lecture claire, avec cartes, textes informatifs et essais d'écrivains de grande qualité et inédits ; un choix de personnages de quartier, vivant et savoureux (la voie tracée par Monsiváis) et une sélection des lieux pas forcément habituelle dans un guide touristique, (comme el Pasaje Pedro Slim, las Peluches Santísima ou le Teatro Blanquita) pour n'en citer que quelques-uns, ce guide, conçu d'abord pour un public mexicain, est aussi un nouvel objet littéraire, comme nous l'avons signalé pour Citámbulos.

Un quatrième guide, ce dernier au format de poche, STYLE MAP CIUDAD DE MEXICO, La guía para conocedores, Lo mejor de CULTURA RESTAURANTES COMPRAS VIDA NOCTURNA ${ }^{53}$ n'a d'autre prétention que celle d'informer le visiteur et de le guider dans la sélection qu'il a faite des quartiers et de leurs offres culturelles et de loisirs. Il apparaît comme un "symptôme " plus modeste mais non moins explicite de ce changement de regard sur la ville et du renouvellement de ses publics. Il est découpé en sept quartiers qui n'ont pas été choisis au hasard, précédés par un texte qui en vante "l'esprit»: quartiers récemment branchés (CENTRO, ROMA, CONDESA); quartiers touristiques (CENTRO, ZONA ROSA); quartiers au haut pouvoir d'achat (POLANCO/REFORMA/LAS LOMAS); nouveaux quartiers d'affaires et résidentiels (SANTA FE) et quartiers anciens pittoresques et recherchés par la bourgeoisie intellectuelle et artistique (SAN ANGEL/ COYOACAN). Ils sont accompagnés de leurs cartes et lieux d'intérêt, des adresses de restaurants, hôtels, magasins et galeries à visibilité internationale. La quatrième de couverture nous indique que Style Map est une invitation pour trouver sa place dans l'une des villes les plus dynamiques, créatives et exceptionnelles du monde (...) Ce guide offre ce qui est le plus en vue de la ville de Mexico pour explorer ses quartiers les plus surprenants, historiques et plein d'énergie (...) Style Map est l'introduction la plus intelligente aux plaisirs de la ville de Mexico.

Style Map est un petit guide facilement manipulable, pour voyageur pressé. Il s'attache à la dimension internationale de la ville et vise un public également international. Son édition en anglais - ce qui est aussi le cas de Citámbulos - met l'accent sur son objectif d'ouverture à un certain type de touristes étrangers. Dans son bref texte d'introduction, DF : ciudad máxima, il est écrit que ${ }^{54}$ Ancienne et en même temps avec un pied dans le futur, la ville de Mexico est reconnue, aimée et fabuleuse; statique et chaotique; ambitieuse et déchirée; altière et humble. C'est la capitale culturelle du Mexique et peut-être de l'Hémisphère occidental. Rien de moins... 


\section{Conclusion} consommation culturelle urbaine, peuvent sembler des questions d'importance relative dans un océan de problèmes urgents à résoudre. Ils permettent néanmoins d'oublier, pour un temps, le désastre ambiant. Ils ouvrent une respiration autant symbolique que visuelle et apparaissent comme une oasis dans le chaos urbain dominant. Pourtant, ce regard sur la ville auquel nous nous sommes attaché en mettant l'accent sur l'apparition de ces nouveaux guides culturels et leur concomitance avec la transformation urbaine a ses limites car la grande majorité des habitants du District Fédéral (D.F.) en est exclue. En effet, il concerne une petite fraction de la population, constituée d'une élite jeune et adulte, éduquée, informée, consommatrice, ayant souvent voyagé à l'extérieur du pays. Quant au Gouvernement de la Ville, son soutien est un autre élément clef de cette situation. En effet, l'opération politique et symbolique que constitue son implication financière et médiatique dans l'édition des nouveaux guides, lui permet de projeter une image séduisante de Mexico, en privilégiant de rares quartiers qui peuvent aussi attirer le tourisme extérieur et focaliser l'attention de tous les publics sur un lieu symboliquement fort dans l'inconscient national, le centre historique. Dans cette étude, nous n'avons pas mesuré l'impact de ces nouveaux guides sur les changements urbains à plus long terme. Dans la mesure où leur diffusion est limitée à quelques élites, nous pouvons nous poser la question de leur influence sur l'évolution d'autres quartiers et sur de nouvelles formes de consommation qui pourraient les accompagner, en incorporant ou en excluant les plus anciennes. 
Ce que partagent, avec passion, les écrivains associés à la rédaction de ces guides.

\section{BIBLIOGRAPHIE}

$\mathrm{ABC} \mathrm{DF}$, diccionario gráfico de la ciudad de México (avec CD rom), ediciones Diamantina, Fundación Televisa, Mexico 2001, 1500p.

¿A donde vaís monsivaís?, Guia del DF de Carlos Monsivaís, ediciones Deborah Holtz, Trilce, Mexico 2010, 358p.

ALGARABÍA, revue mensuelle, direction éditoriale Francisco Masse, ediciones Otras Inquisiciones Mexico DF (environ 120 p.).

CALVINO Italo, Les villes invisibles, éditions Seuil, collection 'Points', Paris 1996.

CITÁMBULOS, El transcurrir de lo insólito, Guía de asombros de la Ciudad de México, Ana Alvarrez, Valentina Rojas Loa, Christian Wissel, ediciones Océano, Mexico 2006, 388p. http:// www.citambulos.net/publicaciones.html

CHILANGO, revue mensuelle, Grupo Expansión, direction éditoriale Gabriela Saïd, Mexico DF (environ 130p.) www.chilango.com/

CHILANGUIA, La ciudad de la cultura, guide mensuel, édition de la Secretaría de Cultura del DF, gratuit, www.cultura.df.gob.mx

Divers auteurs, Rafael Heliodoro Valle, Guillermo Tovar de Teresa, José Lorenzo Cossio, Guía retrospectiva de la Ciudad de México, Segumex, Mexico 1990, 176p.

FERNANDEZ Justino, A guide to Mexican Art, University Of Chicago Press, 1969, 398p.

FRENTE, revue culturelle hebdomadaire créée en mars 2011, direction éditoriale Raúl David Vasquez, publiée par la Ciudad de Frente a sus Contenidos, Mexico DF (gratuit), environ 45 p.).

GALLO Rubén, México DF: lecturas para paseantes, Una antología de Rubén Gallo, Turner Publicaciones, Madrid 2005, 390p.

GRUZINSKI Serge, Histoire de Mexico, éditions Fayard, Paris 1996, 454p.

IBARGÜENGOITIA Jorge, Instrucciones para vivir en México, ediciones Joaquín Mortiz, Mexico 1990, 295p.

JIMENEZ Armando, Sitios de rompe y rasga en la ciudad de México, Salones de baile, cabarets, billares, teatros, ediciones Océano, Mexico 1998, 280p.

LA TEMPESTAD, revue culturelle, 8 numéros par an, Mexico.

LOPEZ GARCÍA Isabelle, Généalogie d'un genre : les chroniques lémébiliennes, Université ParisSorbonne, Paris IV, CRIMIC-SAL 2007 (source Internet).

Nueva guía del centro histórico de México, Nueva guía del CENTRO Histórico de México, Prologue de Guillermo Tovar de Teresa. Introduction de Álfonso Álfaro. Textes de Fabrizio Mejía Madrid, Jorge F. Hernandez, Armando Ramirez, Alejandro Hernandez, Antonio Calera-Grobet, Juan Carlos 
Bautista et Marcela Dávalos. Gobierno del Distrito Federal, Travesías Editores, ed. Mapas, Mexico 2011, 285 p.

PAQUETTE Catherine, Les enjeux complexes de la rénovation du Centre Historique de Mexico, conférence du 25 février 2011, Centre d'Information de l'Urbanisme, Paris 2011 (source Internet). PERAS Delphine, Lire, entretien avec Carlos Fuentes, Paris, ler mars 2009 (source Internet).

PLENN Virgina and Jaime, A guide to Modern Mexican Murals, édiciones Tolteca, Mexico 1963, 162p.

RESIDUAL, Intervenciones artisticas en la ciudad, auteurs divers, Museo Universitario de Arte contemporaneo, Universidad Nacional Autonoma de México, 2010, 287p.

RODRIGUEZ Ana Mónica, La Jornada, Mexico 19 février 2011 (source Internet).

STYLE MAP, Ciudad de México, La guía para conocedores, editorial Travesías, Mexico 2010 , 95p.

ZMVM (Zona Metropolitana del Valle de México), edición Pablo León de la Barra, Mexico 2000 (sans pagination).

\section{NOTES}

1. Entretien avec Delphine Peras, "Lire", Paris 01/03/2009 (source Internet).

2. México DF : lecturas para paseantes, Una antología de Rubén Gallo, Turner publicaciones, Madrid, 2005, 390 pages. Ces textes ont été traduits en français et publiés aux éditions Autrement, Mexico, chroniques littéraires d'une mégalopole baroque, Anthologie de Rubén Gallo, Paris 2007, 278 p.

3. Le Stridentisme, mouvement conçu comme un idéal de modernité, sera la première force dissonante dans le champ culturel, la première avant-garde post-révolutionnaire qui prône une utopie urbaine alors ignorée au Mexique, un « défi au passé et au présent ». Proche du Futurisme italien, ce mouvement qui va être actif jusqu'en 1928 et dont les artistes sont engagés politiquement, défend la modernisation technologique du pays et fait l'apologie de la vitesse, du déplacement avec les nouveaux moyens de transport dont l'avion, des couleurs violentes par le dynamisme des formes, la géométrisation, la perception fragmentée de la réalité contemporaine. Il magnifie aussi la radiodiffusion et la photographie et ses «œuvres reflètent la tentative de recréer formellement la complexité sensorielle de l'atmosphère urbaine contemporaine, avec son bombardement d'information visuelle et auditive ».

4. En décembre 1949, un concours-exposition est organisé à Mexico par le journal l'Excelsior sur le thème " La ville de Mexico interprétée par ses peintres ».

5. Le tableau de Juan O' Gorman gagne le ler Prix du concours de l'Excelsior.

6. Nommé chroniqueur officiel de la Ville de México par le Président Gustavo Diaz Ordaz en 1965, il est considéré par Carlos Monsiváis comme "l'idéologue de l'optimisme bourgeois » (Amor Perdido, 1977, p. 267) qui met son talent au service d'une élite dominante.

7. Où il aborde la période post-révolutionnaire et son impact dans la société mexicaine.

8. C. Monsiváis, A ustedes les consta. Antología de la crónica en México, México D. F., ERA, 2003, [1980]. Cité par Isabelle López García, Généalogie d'un genre : les chroniques lémébéliennes, Université Paris-Sorbonne Paris IV CRIMIC-SAL, 2007 (source Internet).

9. Quelques ouvrages emblématiques de Carlos Monsiváis (jamais traduits en France) : Días de guardar (1971), sur le massacre de Tlatelolco; Amor perdido (1977), sur des figures mythiques du cinéma mexicain, la musique populaire, le syndicalisme, Salvador Novo, la gauche et la politique ; Entrada libre, crónicas de la sociedad que se organiza (1987); Escenas de pudor y liviandad (1988), sur la société du spectacle.

10. C. Monsiváis, A ustedes les consta. Antología de la crónica en México, in Isabelle López García, op.cit. 
11. Nom donné à la bande dessinée au Mexique.

12. Dont le célèbre La noche de Tlatelolco. Testimonios de historia oral, sur le massacre de 1968 qui n'a pas été choisi pour être traduit en français mais en anglais (Etats-Unis).

13. Chroniques dans le journal La Jornada.

14. Chroniques dans le journal Reforma.

15. Chroniques dans le journal Excelsior entre 1969 et 1976. Réunies dans un ouvrage paru sous le titre Instrucciones para vivir en México, Obras de Jorge Ibargüengoitia, éd. Joaquin Mortiz, México 1990, 295 pages.

16. Editions Fayard, Paris 1996.

17. Surtout la génération des écrivains de la Rupture et celle des années 90.

18. Éditions du Seuil, collection 'Points', Paris 1996.

19. Op.cit. p. 32 .

20. Héros anonyme de quartier, masqué, il a été pendant des années le défenseur des pauvres. Une figure mythique urbaine de Mexico.

21. Autre légende urbaine, très active à Puerto Rico et à Mexico, sorte de Bête du Gévaudan latino-américaine accusée de tuer les animaux de ferme en suçant leur sang.

22. Segumex, Mexico 1990, $176 \mathrm{p}$.

23. Qui est membre de l'Asociación de cronistas del distrito Federal qui a aujourd'hui un site important sur internet www.cronistasdf.org.mx et qui donne par Delegación (zones adminstratives de la Ville de Mexico qui correspondent à de grands quartiers) les noms et adresses mails des chroniqueurs avec une courte biographie qui indique leurs qualité et leurs spécificités.

24. Ediciones Océano, Mexico, 280p. (il est réédité).

25. Marchands ambulants ou vendeurs à la sauvette.

26. Au cours de ces dernières quinze années, la scène artistique de la capitale a subi de profonds changements : diffusion internationale de quelques artistes (Gabriel Orozco, Pedro Reyes, Carlos Amorales....) ; création de nouveaux canaux de diffusion avec internet (Soma et Toxico - cultura contemporánea - sponsorisée par la Fundación Jumex); d'espaces alternatifs dans la Colonia Condesa, comme La Panadería (1994-2002) ; dans le Centro Histórico, X’Teresa (1993-), Casa Vecina (2005-), Art Deposit (1996-1998) ; dans le Sud, la Quiñonera (1998-) ; dans la colonia Roma, Border centro cultural, etc...

27. Intellectuels, artistes et touristes fréquenteront la Zona Rosa au cours de ces années.

28. Comme la grande exposition $\mathrm{ABCDF}$ Portraits d'une ville, au Palacio de Bellas Artes à Mexico en 2001, puis à l'Instituto de México à Paris (novembre 2002-février 2003) ; en juin-septembre 2002, à New York au Moma PS1, «La ville de Mexico : an exhibition about the exchange Rates of Bodies and Values "; à Paris, à l'Instituto de México, «Instant City ", de mai à août 2003 ; à Cincinnati au Contemporary Art Center, une exposition « Solo de Mexico City », de Carlos Amorales (septembre 2008 à mars 2009) ; en février-mars 2011, «Les exilés de Mexico » à la Galerie Unam (Québec, Canada) ; ou d'expériences récentes (2010) comme celle qui a donné lieu à l'exposition Residual au MUCA Roma (musée universitaire du quartier Roma) sur l'expérience du recyclage et de la création de jardins dans la ville.

29. Réalisé par le LCM (Laboratorio de la ciudad de México), édicion Pablo Léon de la Barra, México, 2000 (sans pagination).Cet ouvrage se définit comme "un outil pour accéder à l'état actuel et aux processus qui ont modelé la croissance de la ZMVM. Ce volume ne présente pas de solutions, ZMVM est un exercice de visualisation, de sélection et de traduction graphique de l'information qui expose les conditions qui définissent la qualité de vie à Mexico. Dans ces pages, on trouve la superposition de deux paysages distincts : l'un est le paysage objectif des statistiques et des informations comparées; l'autre est une observation graphique confiée à douze artistes contemporains qui habitent la ville ».Onze chapitres traitent de la richesse, de la contamination, de l'eau, de l'éducation, de l'habitat, entre autres... Plusieurs artistes comme Pablo Léon de la 
Barra, Pedro Reyes, Laureana Toledo et Iñaki Bonillas ont réalisé les photographies du sujet pour lequel ils ont été invités. Une maquette très réussie, une mise en page aérée, la ponctuation par les photographies (en dehors des plans, des schémas comparatifs, des statistiques...) donne à cet ouvrage une autre dimension : on entre dans la ville réelle (photographies de façades, d'entrées d'immeubles ou de maisons particulières, d'affiches urbaines (los espectaculares), de scènes insolites, de détails des objets quotidiens... le tout pimenté de clins d'œil et d'humour loufoque (un coffre de voiture ouvert avec la moitié d'une vache) qui permettent de transiter dans la ville qui se met à exister, à vivre sous nos yeux. Ce ne sont pas des photographies qui recherchent l'esthétique, mais qui interpellent l'irrationnel. Cet ouvrage n'est pas un guide - selon la définition orthodoxe - , mais de fait en devient un, à la fois très spécialisé, informatif et riche en surprises iconographiques.

30. Ouvrage (accompagné d'un CD Rom) Ed. Diamantina, Fundación Televisa, avec l'appui du Fonca et de Conaculta, Mexico 2001, préface de Cristina Bremer, 1500 pages, 515 entrées, 2000 images, 45 textes et 250 artistes, photographes et écrivains. Le glossaire d'images de A à Z comporte un texte court, des photographies et des textes. Que dit l'introduction? «Un livre sur l'expérience de la vie dans le District Fédéral» qui présente «les multiples formes de l'attachement qu'inspire cette ville-capitale, sur ses aspects les plus intimes et les plus affectueux ».La mise en page fait alterner des photographies de la vie quotidienne du DF comme une boulangerie, un étal de poisson... avec des photographies de détails insolites, des photos ou plan ancien de la ville, des vues d'avion, des gros plans, des portraits dans la rue de hippies, des accumulations (bouteilles, chaises, jouets...) pour n'en citer que quelques-uns. Des textes d'Habermas, Eric Hazan, Alan Riding, Carlos Monsiváis, Carlos Fuentes, Juan Villoro ou Jorge Volpi ; des images de Francis Alÿs et Gabriel Orozco; des photographies de Lourdes Almeida, Mariana Yampolsky, Mélanie Smith ou Lourdes Grobet... Quelques ressorts visuels du livre : la multiplication, les associations, les accumulations, les détails architecturaux répétitifs : dessins de béton, façades, portes, bouches d'égouts et d'eau etc... Des photographies insolites, de détails de visages, de graffiti urbain...Plus qu'un dictionnaire, c'est un inventaire graphique riche, qui fait apparaître ce qui est surprenant, grotesque et le hisse à la catégorie esthétique.

31. Appartenant au PRD (Partido de la Revolución democrática, coalition de gauche modérée). Depuis 2000, quatre Etats de la République mexicaine ont eu des gouverneurs de gauche: Zacatecas, Michoacán, Chiapas et Baja California Sur.

32. Op. cit. p. 40.

33. Cité par Ruben Gallo, op.cit.

34. Op. cit. p. 107.

35. Depuis les années 70 une belle revue mensuelle, Mexico desconocido, fait office de guide pour tout le pays.

36. C'est le nom d'argot de l'habitant du Distrito Federal (DF) et surtout de la ville de Mexico. Bien qu'un peu péjoratif, il est néanmoins revendiqué en général par la population.

37. Sur Internet, on peut lire (extrait) : « ¿Quienes somos? (Qui sommes-nous ?) Depuis 1998, la mission de La Tempestad «a été d'amener l'art au lecteur. Sa maquette reconnaissable, son architecture éditoriale et ses contenus de grande qualité lui ont fait gagner un espace singulier dans la conscience des lecteurs mexicains ».

38. Edité en version électronique depuis quelques mois.

39. «Libro, atlas, manifiesto, almanaque, inventario, objeto inclasificable y heterogéneo como la ciuda de la que da cuenta, esto es, ante todo, una guía para perderse en México.Porque basta salir a la calle, afinar la mirada y abrir todos los sentidos para descubrir que lo insólito - esa delgada línea que corre paralela a la rutina y atraviesa lo inusual, misterioso, exótico, terrible o poético de la ciudad - es también una forma de conocer su esencia.Esa guía es, ante todo, una invitación a abandonar el sillón de lectura, deambular por la ciudad y convertirse en citámbulo dejando que 
los viejos bostezos y muecas de desesperación se transforman en refrescantes bocanadas de asombro».

40. «La primera guía se usa como cualquier otra: seis zonas que cubren toda la mancha urbana de la ZMVM; en cada una de las cuales podrá encontrar itinerarios integrados por lugares geograficamente cercanos. El habitante elegirá uno de ellos, lo recorrerá, tomará fotos de sus lugares favoritos y se las enseñará a su vecino. La segunda guía traza caminos que sorprenden por los vínculos que se establecen entre zonas muy disímiles. Así, al final de cada lugar existen puertas que conducen a uno o varios lugares que, aunque alejados geograficamente, estan conectados tematicamente. La tercera guía es más bien una metaguía, es decir una invitación a ir más allá de lo que deja una relación meramente intuitiva con la ciudad. Asi, un personaje que la habita, pero sobre todo que la estudia, abre paréntesis dentro de los paréntesis aquí sugeridos ». 41. L'arpenteur de la ville.

42. Cité p. 54: «Las lecciones se obtienen no en la contemplación de montañas, árboles o rebaños, sino en el domeñamiento de semáforos y avenidas ".

43. Monsiváis es la ciudad de México, las líneas de su mano son una Guia Roji. La Ciudad de México no existiría sin Monsiváis, quien la engrandece, la hace posible, le ve el lado ciudadano, aún en medio de las ruinas del terremoto de 1985.

44. Ils étaient près de 25000 , certains sont partis, beaucoup sont restés ou se sont déplacés ailleurs.

45. Il existe depuis environ cinq ans et concerne les rues Regina, San Jerónimo et Mesones. Il a été créé et financé par le secteur public, le Gouvernement Fédéral, et l'apport de capitaux privés (Fundación del Centro Histórico).

46. Les enjeux complexes de la rénovation du Centre historique de Mexico, Catherine Paquette, conférence du 25 février 2011, Centre d'information sur l'Urbanisme, Paris.

47. «Los sedimentos del tiempo han dado al centro, a la ciudad y al país, un recurso de esa naturaleza: un recinto que logra integrar las presencias emblemáticas de los múltiples vectores culturales del país, pasados y presentes, reales e imaginarios, activos y latentes. Ni siquiera el más visionario arquitecto barroco hubiera podido imaginar un dispositivo espacial más eficaz para representar a una sociedad heterogénea en busca de armonía, una abigarrada multitud de ciudadanos ansiosos de construirse como nación ».

48. Dans un article de la Jornada du 19 février 2011, les sous-titres de l'article sont les suivants : «Le GDF publie un outil pour rapprocher les Mexicains du cœur de leur capitale » et «Un nouveau guide détaille la vie, l'histoire et la métamorphose du Centre historique ».

49. La Jornada, 19 Février 2011, Mexico (Ana Mónica Rodriguez).

50. La Jornada, idem.

51. «El Zócalo es donde todo comenzó, es el bing bang de concreto. Desde ahi, donde suponemos que estaba el nopal despuès de que el águila volo con la serpiente en el pico, se extendio, como una alfombra roja, el país entero. Ha sido mercado, jardín, plancha, tablero de ajedrez, plato para rosca de Reyes, cine, museo nomada, clínica con mamografias gratis, motel de maestros, aire para volar papalotes, escaparate de vendedores de pastillas de piel de serpiente, pista de hielo, museo de fotografías de niños con elefantes, cementerio con ofrendas para los difuntitos, sala de conciertos, teatro donde el mejor espectáculo es la propia multitud que se mira en su inmensidad (...) El Zócalo es la definición de lo chilango : todo cabe, nada sobra ».

52. Tambour de bois du nom de l'arbre, l'ahuehuete, qui sert à fabriquer l'instrument.

53. Format de poche $(10,5 \times 15 \mathrm{~cm})$, textes non signés, Editorial Travesías, Mexico 2010, 95 pages (versions en espagnol et anglais.

54. «Antigua y al mismo tiempo con un pie en el futuro, la ciudad de México es notoria, amada y fabulosa; estatica y caótica, ambiciosa y despedazada ; altiva y humilde. Es la capital cultural de México y tal vez del hemísfero occidental ». 


\section{RÉSUMÉS}

Au Mexique, les discours sur la ville et l'imaginaire urbain vont s'accompagner, au tournant des années 2000, de formes d'expression inédites liées à de nouveaux supports éditoriaux. C'est toute la conception du statut de la ville comme objet touristique qui va changer au cours des deux dernières décennies, passant de la tradition du «tourisme extérieur » à la pratique jouissive du «tourisme intérieur ", la « périphérie » se déplaçant alors vers le «centre historique ». Les trois principaux guides touristiques que nous avons choisi de commenter (CITÁMBULOS Guía de asombros Ciudad de México. El transcurrir de lo insólito) ; ¿A DÓNDE VÁIS MONSIVÁIS ? Guía del DF de Carlos Monsiváis; et Nueva guía del CENTRO Histórico de México), s'inscrivent dans le développement de ces nouveaux discours et récits sur la ville, en dehors de la place qu'y occupe la littérature actuelle dont nous ne parlerons pas ici. Différents dans la forme, le format et le fond, ils sont chacun les héritiers de cette façon plus libre de chercher une autre ville dans la ville, et de vivre sa ville.

A principios del siglo XXI, los discursos sobre la ciudad y el imaginario urbano mexicanos incluyen formas de expresion inéditas, ligadas a nuevos soportes editoriales. Toda la concepción del estatuto de la ciudad como objeto turístico cambiará en el transcurso de las dos últimas decadas, pasando de la tradición del «turismo exterior» al gozo del «turismo interior», desplazando la "periferia» hacia el «centro histórico». Las tres principales guías turísticos que hemos seleccionado (CITÁMBULOS Guía de asombros de la Ciudad de México. El transcurrir de lo insólito); ¿A DÓNDE VÁIS MONSIVÁIS? Guía del DF de Carlos Monsiváis; y Nueva guía del CENTRO Histórico de México), se inscriben en el desarrollo de estos nuevos discursos y relatos sobre la ciudad, fuera del lugar que ocupa la literatura actual, de la cual no hablaremos aqui. Diferentes por su forma, su formato y su contenido, cada una es heredera de esta nueva manera más libre de buscar otra ciudad en la ciudad, y de vivir su ciudad.

\section{INDEX}

Mots-clés : Chilango, Monsiváis, centre historique, guide, chronique, Condesa, Roma, culture et imaginaires urbains, "ville invisible », tourisme intérieur, déambulation

\section{AUTEUR}

\section{CHRISTINE FRÉROT}

Historienne d'art et critique d'art. Ehess/Cral 